\title{
Le laser à électrons libres UV de super-ACO : source et applications
}

\author{
L. Nahon ${ }^{1,2}$, E. Renault ${ }^{1,2}$, D. Nutarelli ${ }^{1}$, D. Garzella ${ }^{1,2}$, G. De Ninno ${ }^{1,2}$, M. Hirsch ${ }^{1,2}$ \\ et M.E. Couprie ${ }^{1,2}$ \\ ${ }^{1}$ LURE, bâtiment 209D, Université Paris Sud, BP. 34, 91898 Orsay cedex, France \\ 2 SPAM/DRECAMIDSM, CE de Saclay, bâtiment 522, 91191 Gif-sur-Yvette cedex, France
}

\begin{abstract}
Résumé : Les lasers à électrons libres (LEL) sur anneau de stockage sont des sources cohérentes, accordables et intenses qui peuvent couvrir le domaine UV, comme celui de Super-ACO qui permet d'atteindre $300 \mathrm{~nm}$ avec des puissances et des niveaux de stabilité très satisfaisants. Ces performances ont permis d'initier un programme d'applications basé notamment sur des expériences pompe/sonde résolues en temps réalisées en combinant le LEL avec le Rayonnement Synchrotron, émis par différentes lignes de lumière, et naturellement synchronisé. Le champ d'application s'étend de la physique des surfaces, aux sciences des matériaux et à la photo-biologie en solution.
\end{abstract}

\section{LA SOURCE LEL}

Basé sur le principe de l'interaction entre une onde lumineuse stockée dans une cavité optique et des paquets d'électrons traversant le champ magnétique sinusoïdal d'un onduleur, les Laser à Electrons Libres (LELs) possèdent des propriétés uniques en terme d'accordabilité potentielle et de puissance moyenne. C'est notamment le cas des LELs sur anneaux de stockage (cf figure1) qui de par la haute énergie des particules stockées et la qualité du faisceau, permettent d'atteindre le domaine UV. Ainsi le LEL de l'anneau SuperACO du Lure $(800 \mathrm{MeV})$, oscille depuis le visible jusqu'à $350 \mathrm{~nm}$ depuis 1990 , et depuis l'année demière autour de $300 \mathrm{~nm}$ [1], avec les puissance moyenne les plus élevées, jusqu'à $300 \mathrm{~mW}$ et une accordabilité de quelques dizaines de nm pour un jeu de miroirs donnés.

Le comportement du laser est régi par la condition de seuil (gain > pertes), ce qui nous a conduit, au vu du faible gain accessible (autour de $2 \%$ ) à entreprendre un travail important de minimisation des pertes (absorption, diffusion, transmission) via la caractérisation des miroirs multi-couches qui composent la cavité, et qui sont soumis aux conditions extrêmes associées au rayonnement synchrotron de l'onduleur : charge thermique élevée et spectre X-UV entrainant des dégradations de nature photochimique [2].

Par ailleurs, la stabilité du laser est un paramètre critique, qui relève d'une dynamique complexe abordée tant par des simulations numériques, que par des mesures de durée des impulsions laser et du paquets d'électrons, dont les évolutions sont liées. De plus, une contre-réaction longitudinale agissant sur le laser lui-même a permis de stabiliser le laser, de façon très satisfaisante, à la fois en terme de puissance moyenne (fluctuations inférieures à $1 \%$ pendant $1 \mathrm{~h})$ et d'affinement spectral $(\Delta \lambda=0.3 \AA$ ) et temporel $(\Delta t$ $\sim 20$ ps) permettant d'approcher la limite de Fourier [3].

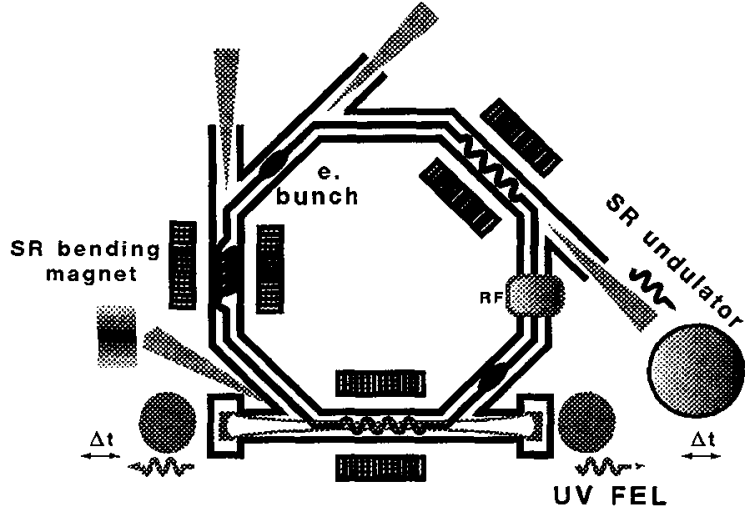

Figure 1: Diagramme d'implantation d'un LEL sur ameau de stockage illustrant ses possibilités de couplage avec le rayonnement synchrotron issu de différents types de lignes de lumière. 


\section{LE PROGRAMME D'APPLICATION}

Fort de ces performances, le LEL de Super-ACO, source cohérente, intense et accordable dans l'UV, a atteint un stade de maturité telle que depuis 1993, un programme d'application soutenu a été mis en place mené parallèlement au développement de la source elle-même. Après une expérience à un photon LEL de déclin de fluorescence polarisée en biologie [4], les applications se sont portées sur des expériences pompe/sonde à deux couleurs combinant le LEL et le Rayonnement Synchrotron (RS). Grâce à la synchronisation naturelle entre les deux sources, et à leur accordabilité, on peut effectuer des expériences résolues en temps et/ou en fréquence adaptées à l'études d'états excités et plus généralement de systèmes hors d'équilibre [5].

Les expériences développées à ce jour concernent différents champs scientifiques :

(i) En physique des surfaces, le LEL est utilisé pour exciter des porteurs de charges aux interfaces de semi-conducteurs induisant une modifications transitoire de la structure de bandes électroniques. Ces mouvements sont suivis à l'échelle sub-ns par photoémission de coeur à l'aide du RS. Dans le cas de la surface de $\operatorname{Si}(111) 2 \times 1$, nous avons ainsi pu mettre en évidence une dynamique de relaxation particulière faisant intervenir explicitement les états de surface dans les premières centaines de ps suivant l'excitation LEL [6]. D'autres interfaces comme Si/SiO2, aussi étudiée, présentent des comportement différents en fonction de l'épaisseur de recouvrement d'oxyde, mettant en évidence des effets d'accumulation de charge à la surface de la couche d'oxyde [7].

(ii) En sciences des matériaux, nous avons développé une expérience pompe/sonde LEL + RS dans laquelle on utilise le LEL pour exciter un système dont la dynamique de relaxation est sondée par absorption IR par transformée de Fourier, éventuellement associée à un microscope grâce au continuum blanc dans l'IR foumi par un aimant de courbure (visible $-20 \mu \mathrm{m}$ ). Ayant pu observer la synchronisation des deux sources au foyer du microscope, nous avons suivi les modifications dans la structure vibrationnelle d'un minéral (la kaolinite) irradié par le LEL, mettant en évidence la photodésorption induite par le LEL de groupements chimiques $\mathrm{OH}$ externes [8]. Des expériences à l'échelle de la ns sont prévues sur des films minces ainsi que sur des molécules en solution.

(iii) En bio-chimie, nous avons initié une expérience d'absorption transitoire consistant à utiliser le continuum UV-visible du RS issu d'un aimant de courbure pour sonder la dynamique de relaxation, et les espèces fugitives associées, suite à l'absorption d'un photon LEL-UV par un chromophore en solution. La faisabilité en terme de flux a été démontrée sur une molécule test le POPOP, utilisé comme colorant-laser [9]. Les premiers résultats portant sur la dynamique de relaxation à l'échelle ns ont été récemment obtenus sur une autre molécule, l'acridine, chromophore d'intérêt biologique [10].

L'ensemble de ces activités, tant au niveau du développement de la source que de son programme d'applications, s'inscrit dans la perspective du LEL de SOLEIL, une source ps accordable dans le domaine UV/VUV présentant des performances sans équivalents, notamment en terme de flux moyen.

\section{Références}

1. D. Nutarelli, D. Garzella, E. Renault, L. Nahon and M.E. Couprie, NIM A 445, 143 (2000).

2. D. Nutarelli, Thèse de Doctorat, Université Paris-Sud (2000).

3. M.E. Couprie, D. Nutarelli, R. Roux, L. Nahon, B. Visentin, A. Delboulbé, G. Flynn et M. Billardon, NIM A 407, 215 (1998).

4. M.E. Couprie, F. Mérola, P. Tauc, D. Garzella, A. Delboulbé, T. Hara et M. Billardon, Rev. Scient. Instrum. 65, 1485 (1994).

5. L. Nahon, E. Renault, M.E. Couprie, F. Mérola, P. Dumas, M. Marsi, A. Taleb-Ibrahimi, D. Nutarelli, R. Roux et M. Billardon, NIM A 429, 489 (1999).

6. M. Marsi, M.E. Couprie, L. Nahon, D. Garzella, A. Delboulbé, T. Hara, R. Bakker, G. Indlekofer, M. Billardon et A. Taleb-Ibrahimi, Appl. Phys. Lett. 70, 895 (1997).

7. M. Marsi, R. Belkou, C. Grupp, G. Panacione, A. Taleb-Ibrahimi, L. Nahon, D. Garzella, D. Nutarelli, E. Renault, R. Roux, M.E. Couprie et M. Billardon, Phys. Rev. B 61, R5070 (2000).

8. L. Nahon, E. Renault, M.E. Couprie, D. Nutarelli, D. Garzella, F. Polack, G. L. Carr, G. Williams et P. Dumas, Proc. SPIE 3775, 145 (1999).

9. E. Renault, L. Nahon, D. Nutarelli, D. Garzella, F. Mérola et M.E.Couprie, Proc. SPIE 3925, 29 (2000).

10. E. Renault, L. Nahon, D. Garzella, G. De Ninno, M. Hirsch et M.E. Couprie, NIM A (sous presse). 